\title{
ON THE REGULARITY OF MEASURES ON LOCALLY COMPACT SPACES ${ }^{1}$
}

\author{
MARK LEVIN ${ }^{2}$ AND WILBUR STILES
}

\begin{abstract}
The purpose of this paper is to present the two following theorems:

(1) Every Baire measure on the $\sigma$-algebra $\mathscr{B}_{a}$ generated by the compact $\mathscr{G}_{\delta}$ subsets of a paracompact, locally compact space is outer regular; (2) in a paracompact, locally compact space, any Baire measure on $\mathscr{B}_{a}$ can be extended to an outer regular Borel measure on the $\sigma$-algebra generated by the closed subsets.

In addition, this paper contains an example which shows that neither of these two theorems is true for all arbitrary locally compact Hausdorff spaces.
\end{abstract}

1. Introduction. A standard theorem of measure theory states that every Baire measure on a locally compact Hausdorff space $X$ is both inner and outer regular on the $\sigma$-ring $\mathscr{R}_{a}$ generated by the compact $\mathscr{G}_{\delta}$ subsets of $X$ (cf. [4]). Another standard theorem states that every Baire measure on $\mathscr{R}_{a}$ can be extended to a Borel measure on the $\sigma$ algebra $\mathscr{B}_{0}$ generated by the closed subsets of $X$. The purpose of this note is (1) to show that if the space is paracompact and the Baire measure is inner regular on $\mathscr{B}_{a}$ (the $\sigma$-algebra generated by the compact $\mathscr{G}_{\delta}$ subsets of $X$ ), then the Baire measure can be extended to a Borel measure on $\mathscr{B}_{0}$ so that its extension is also inner and outer regular, and (2) to show that (without the assumption of paracompactness) such an extension is not always possible.

2. Definitions. In the following, $X$ will be a locally compact Hausdorff space. A subset of $X$ will be called a Baire set (Borel set, resp.) if and only if it is a member of the smallest $\sigma$-algebra $\mathscr{B}_{a}\left(\mathscr{B}_{0}\right.$, resp.) containing all the compact $\mathscr{G}_{\delta}$ sets (closed sets, resp.). A measure $\mu$ on $\mathscr{B}_{a}\left(\mathscr{B}_{0}\right.$, resp.) will be called a Baire (Borel, resp.) measure if it is finite on all the compact

Received by the editors August 17, 1971 and, in revised form, January 3, 1972.

AMS 1969 subject classifications. Primary 2813; Secondary 2830.

Key words and phrases. Baire measure, Borel measure, outer regular measure, locally compact space, paracompact space.

1 Supported by contract 011328000 .

2 This author's work was supported in part by the National Science Foundation (NSF GP-9632).

(c) American Mathematical Society 1972 
$\mathscr{G}_{\delta}$ sets (compact sets, resp.). A Baire (Borel, resp.) measure will be called inner regular if

$$
\mu(E)=\sup \left\{\mu(K): K \subset E, K \text { compact } \mathscr{G}_{\delta}(K \text { compact, resp. })\right\}
$$

for all Baire (Borel, resp.) sets E. A Baire (Borel, resp.) measure will be called outer regular if

$$
\mu(E)=\inf \left\{\mu(0): 0 \supset E, 0 \text { open, and } 0 \in \mathscr{B}_{a}(0 \text { open, resp. })\right\}
$$

for all Baire (Borel, resp.) sets E. A Baire (Borel, resp.) measure will be called regular if it is both outer regular and inner regular.

A set will be called $\sigma$-compact if it is the union of countably many compact sets; $\sigma$-bounded if it is contained in a $\sigma$-compact set.

3. Regularity of Baire measures. Problem 14.10b of [6] states (after a suitable adjustment of terminology) that every inner regular Baire measure is outer regular. The author of [6] then uses this result to prove Theorem 14.11 which asserts that any inner regular Baire measure can be extended to an outer regular Borel measure. An example will be given in $\S 5$ to show that these results are not correct. We now show that the first of these results is correct if the space is Hausdorff and paracompact. (We are indebted to the referee for having first pointed out to us that this result is true in a metrizable space.)

THEOREM 1. Any Baire measure $\mu$ on a locally compact paracompact Hausdorff space $X$ is outer regular.

Proof. Let $E$ be a member of $\mathscr{B}_{a}$. We wish to show that $\mu(E)=$ $\inf \left\{\mu(0): 0 \supset E, 0\right.$ open, $\left.0 \in \mathscr{B}_{a}\right\}$. This is known (Proposition 14.6 of [6]) when $E \in \mathscr{R}_{a}$ and trivial when $\mu(E)=\infty$. Hence we may assume that $\mu(E)<\infty$ and that $A=X \backslash E$ is $\sigma$-bounded. Since $X$ is paracompact and locally compact, $\bar{A}$ is $\sigma$-bounded; so, by the Baire sandwich theorem, $\bar{A}$ is contained in a countable union $B$ of open subsets of $\mathscr{R}_{a}$. A paracompact space is normal; so, by Urysohn's lemma, there exists a continuous function $f$ such that $f(x)=1$ for all $x$ in $\bar{A}$ and $f(x)=0$ for all $x$ in $X \backslash B$. Let $C=\{x: f(x)=1\}$. $C$ is a closed $\mathscr{G}_{\delta}$ which contains $\bar{A}$, and, since $C \subset B, C$ is also $\sigma$-bounded. Hence, given any $\varepsilon>0$ there exists an open Baire set $G$ containing $C \cap E$ such that $\mu(G)<\mu(C \cap E)+\varepsilon$. Then $G \cup$ $(X \backslash C)$ is an open Baire set containing $E$, and since $X \backslash C=E \backslash C$,

$$
\begin{aligned}
\mu(G \cup(X \backslash C)) & \leqq \mu(G)+\mu(X \backslash C) \\
& <\mu(C \cap E)+\varepsilon+\mu(E \backslash C) \leqq \mu(E)+\varepsilon .
\end{aligned}
$$

The preceding theorem allows us to prove the following. 
Proposition. Let $X$ be a metrizable or $\sigma$-compact locally compact Hausdorff space which is not compact. Then for any nonnegative extended real number $\alpha$, there exists a regular Baire measure $\mu$ on $X$ such that $\mu(X)=\alpha$.

ProOF. Let $\left\{\alpha_{n}\right\}_{n=1}^{\infty}$ be a sequence of nonnegative real numbers such that $\alpha=\sum_{n} \alpha_{n}$. If $X$ is metrizable, let $\left\{x_{n}\right\}_{n=1}^{\infty}$ be a sequence of distinct elements of $X$ which has no cluster point. Define a measure $\mu$ on $\mathscr{B}_{a}$ such that $\mu(E)=\sum\left\{\alpha_{n}: x_{n} \in E\right\}$ for each $E \in \mathscr{B}_{a}$. A routine examination of $\mu$ shows that $\mu$ is, in fact, an inner regular Baire measure on $X$ and $\mu(X)=\alpha$. Theorem 1 guarantees that $\mu$ be regular. If $X$ is $\sigma$-compact, let $\left\{K_{n}\right\}_{n=1}^{\infty}$ be a sequence of compact $\mathscr{G}_{\delta}$ subsets of $X$ such that $\operatorname{int}\left(K_{n}\right)$ is a Baire set, $\operatorname{int}\left(K_{n+1}\right) \varsubsetneqq K_{n}$, and $X=\bigcup_{n} K_{n}$ for $n=1,2, \cdots$. Let $C_{n}=K_{2 n} \backslash\left(\operatorname{int}\left(K_{2 n-1}\right)\right)$ for $n=1,2, \cdots$. Then $C_{n}$ is a nonempty compact Baire set. Let $\mu_{n}$ be any Baire measure on $C_{n}$ such that $\mu_{n}\left(C_{n}\right)=\alpha_{n}$. Define a measure $\mu$ on $\mathscr{B}_{a}$ such that $\mu(E)=\sum_{n} \mu_{n}\left(E \cap C_{n}\right)$ for each $E \in \mathscr{B}_{a}$.

Once again, a routine examination shows that $\mu$ is a (regular) Baire measure such that $\mu(X)=\alpha$.

COROllaRY. Let $X$ be a metrizable or o-compact locally compact Hausdorff space. If $X$ is not compact, then there exists a discontinuous positive linear functional on $C_{0}(X)$, the normed space of continuous realvalued functions on $X$ with compact support.

The proof of this corollary follows immediately from the Riesz representation theorem. One might naturally conjecture that a similar theorm holds for all $C_{0}(X)$. This however is not true. In fact, there exist examples of noncompact locally compact spaces $X$ for which every positive linear functional on $C_{0}(X)$ is continuous or, equivalently, for which $\mu(X)<\infty$ for all inner regular Baire measures $\mu$ on $X$. A particular example with this property can be obtained from the topological space $X$ of all ordinals less than the first uncountable ordinal furnished with the order topology. Every Baire measure on $X$ is finite because every monotonic real-valued function on $X$ is eventually constant.

As the following example shows, the analogue for Borel measures of Theorem 1 is not true. Let $Y$ be the Euclidean plane. Define a topology on $Y$ such that a subset $E$ of $Y$ is open if and only if the intersection of $E$ with each vertical line $L$ is an open set in $L$ with respect to the usual topology of the line. The resulting space is a metrizable locally compact space, and we can define an inner regular Borel measure $\mu$ on this space by letting $\mu(E)=\sum_{L} \lambda(E \cap L)$ where $\lambda$ is Lebesgue linear measure. If $R$ denotes the horizontal axis, then $R$ is a Borel set and $\mu(R)=0$. However, $\mu(0)=\infty$ for every open set, 0 , containing $R$. 
Even the presence of metrizability is not enough to imply that (outer regular) Baire measures are inner regular or that outer regular Borel measures are inner regular. The first of these claims can easily be seen by considering the following example: Let $X$ be an uncountable set with the discrete topology, and let $\nu$ be the measure which is zero on countable sets and one on their complements. Then $v$ is a finite Baire measure which is outer regular but is not inner regular. The second of these claims can be seen by changing the measure $\mu$ in the space $Y$ given above so that the new measure agrees with $\mu$ on sets which are contained in a countable union of vertical lines and is infinity otherwise.

4. Extension of Baire measures. Since every finite inner regular Baire measure is outer regular, we can prove the following by using Theorem 1 .

THEOREM 2. Let $\mu$ be an inner regular measure on a locally compact Hausdorff space $X$. If $\mu$ is finite or if $X$ is paracompact, then there exists a unique Borel measure $\bar{\mu}$ on $\mathscr{B}_{0}$ which extends $\mu$ such that:

(i) For each open set $0, \tilde{\mu}(0)=\sup \left\{\mu(K): K \subset 0, K\right.$ compact $\left.\mathscr{G}_{\delta}\right\}$.

(ii) For each Borel set $E, \bar{\mu}(E)=\inf \{\bar{\mu}(0): E \subset 0,0$ an open set $\}$.

The measure $\bar{\mu}$ also satisfies the following:

(iii) For each Borel set $E$ with $\bar{\mu}(E)<\infty, \bar{\mu}(E)=\sup \{\bar{\mu}(K): K \subset E, K$ compact $\}$.

(iv) For each Borel set with $\bar{\mu}(E)<\infty$ there is a Baire set $H$ and a Borel set $N$ with $\bar{\mu}(N)=0$ and $E=H \Delta N$.

(v) If $\mu$ is finite, then $\bar{\mu}$ is finite. Hence, in this case, $\bar{\mu}$ is regular.

If $v$ is any Baire measure defined on $\mathscr{R}_{a}$, then $v$ has an extension to $\mathscr{B}_{0}$ such that (i)-(v) hold.

Proof. Since most of the pertinent details are contained in Theorem 14.11 of [6], we merely sketch the proof and indicate the appropriate modifications. Define $\mu^{*}$ for each open set 0 by

$$
\mu^{*}(0)=\sup \left\{\mu(K): K \subset 0, K \text { compact } \mathscr{G}_{\delta}\right\} .
$$

Let $\mathscr{M}=\{M \subset X$ : given $\varepsilon>0$, there exists an open set 0 such that $0 \supset M$ and $\left.\mu^{*}(0)<\varepsilon\right\}$. Then $\mathscr{A} \equiv\left\{H \triangle M: H \in \mathscr{B}_{a}, M \in \mathscr{M}\right\}$ is a $\sigma$-algebra. Let $\mu_{1}(H \triangle M)=\mu(H)$ for $H \triangle M \in \mathscr{A}$. Then $\mu_{1}$ is a measure on $\mathscr{A}$. Finally, let $\mathscr{B}$ be the set of all locally measurable sets with respect to $\mathscr{A}$, and if $B \in \mathscr{B}$, let $\bar{\mu}(B)=\mu_{1}(B)$ if $B \in \mathscr{A}$ and let $\bar{\mu}(B)=\infty$ otherwise. Then $\mathscr{B}$ contains $\mathscr{B}_{0}$ and $\bar{\mu}$ restricted to $\mathscr{B}_{0}$ satisfies the properties given in the theorem.

The final assertion of the theorem can be verified by applying the above procedure to $\mathscr{R}_{a}$ even if $v$ is not finite or $X$ is not paracompact. If $v$ is finite or if $X$ is paracompact, then, alternatively, $v$ can be extended to an 
inner regular measure on $\mathscr{B}_{a}$, and the above procedure can be applied to $\mathscr{B}_{a}$.

5. Example. We conclude by constructing an example of a Baire measure $\mu$ defined on $\mathscr{B}_{a}$ which is inner regular but not outer regular. $\mu$ also has the following property: If $\bar{\mu}$ is any Borel measure on $\mathscr{B}_{0}$ which agrees with $\mu$ on $\mathscr{B}_{a}$, then $\bar{\mu}$ is not outer regular. This last property makes it clear that $\mu$ cannot be extended from $\mathscr{B}_{a}$ to $\mathscr{B}_{0}$ in such a way that assertion (ii) of Theorem 2 holds. Hence the statement of Theorem 2 requires some hypotheses such as finiteness of the measure or paracompactness of the space. This shows, of course, that Theorem 14.11 of [6] is not correct. The example also shows that Problem 14.10b of [6] is not correct. Our example is constructed as follows.

Let $D$ be the set of all dyadic rational numbers, and let $E=R \backslash D$ be the complement of this set in the set of real numbers $R$. For each nonnegative integer $n$ and for each integer $j$, let $D_{n, j}$ be the interval $\left[j / 2^{n},(j+1) / 2^{n}\right]$. If $j$ is an integer, let $\hat{D}_{n, j}$ be the singleton $\left\{D_{n, j}\right\}$. Let $X$ be the space whose points are elements of $E \cup\left(\left\{\hat{D}_{n, j}: n, j\right.\right.$ integers and $\left.\left.n \geqq 0\right\}\right)$. We topologize $X$ as follows. If $x \in E$ and $N \subset X$ contains $x$, then $N$ is a neighborhood of $x$ exactly when $N$ contains all but finitely many of the $\hat{D}_{n, j}$ 's for which $x \in\left[j / 2^{n},(j+1) / 2^{n}\right]$. If $y=\hat{D}_{n, j}$, then any set containing $y$ is a neighborhood of $y$. It is not difficult to see that $X$ becomes a locally compact Hausdorff space when the neighborhoods are defined as above and it is also not difficult to see that every subset of $X$ is a Borel set and the Baire sets are those subsets of $X$ which are countable or cocountable. We define a measure $\mu$ on the Baire sets of $X$ such that for each $\hat{D}_{n, j}, \mu\left(\left\{\hat{D}_{n, j}\right\}\right)=1 / 2^{n}$ and for each Baire set $B, \mu(B)=\sum \hat{D}_{n, j} \in B \quad \mu\left(\left\{\hat{D}_{n, j}\right\}\right)$. It is easy to see that $\mu$ is indeed a Baire measure; furthermore, it is also easy to see that $\mu$ is inner regular, $E$ is a Baire set and $\mu(E)=0$. To complete the example, we need only show that

$$
\mu^{*}(0)=\sup \left\{\mu(K): K \subset 0, K \text { compact } \mathscr{G}_{\delta}\right\}=\infty
$$

for each open subset 0 containing $E$. So, we let 0 be any open set containing $E$, and we let

$$
E_{n}=\left\{x \in E: x \in D_{m, j} \text { and } \hat{D}_{m, j} \in 0 \text { for all } m \geqq n \text { for some } j\right\} \text {. }
$$

Since $E=\bigcup_{1}^{\infty} E_{n}$, it must be true that the Lebesgue outer measure $\lambda^{*}$ of some $E_{n}$ is not zero. Suppose $\lambda^{*}\left(E_{n_{0}}\right)>0$. Then, for each $m \geqq n_{0}$, $\lambda^{*}\left(\bigcup_{j}\left\{D_{m, j} \hat{D}_{m, j} \in 0\right\}\right) \geqq \lambda^{*}\left(E_{n_{0}}\right)$. Hence for each $m \geqq n_{0}, \mu\left(0 \cap X_{m}\right) \geqq$ $\lambda^{*}\left(E_{n_{0}}\right)$ where $X_{m}=\left\{\hat{D}_{m, j}: j\right.$ is an integer $\}$. This implies that $\mu^{*}(0)=\infty$. Thus, for any Borel extension $\bar{\mu}$ of $\mu, \bar{\mu}(0)=\infty$ for any open set 0 containing $E$. 
In closing, we note that the regularity of measures has also been investigated by S. Berberian in [1], [2], and [3], and by K. Ross and K. Stromberg in [5].

\section{REFERENCES}

1. S. K. Berberian, Counterexamples in Haar measure, Amer. Math. Monthly 73 (1966), no. 41, part II, 135-140. MR 33 \#4180.

2. - Sesquiregular measures, Amer. Math. Monthly 74 (1967), 986-990. MR 36 \#2766.

3. - On the extension of Borel measures, Proc. Amer. Math. Soc. 16 (1965), 415-418. MR 31 \#298.

4. P. R. Halmos, Measure theory, Van Nostrand, Princeton, N.J., 1950. MR 11, 504.

5. K. A. Ross and K. Stromberg, Baire sets and Baire measures, Ark. Mat. 6 (1965), 151-160. MR 33 \#4224.

6. H. L. Royden, Real analysis, Macmillan, New York, 1968.

Department of Mathematics, Florida State University, Tallahassee, Florida 32306 